\title{
Significance of Internal Working Models Based on Psychopathology
}

\author{
Li Tianwei $^{1, a}$, Li Fuyuan ${ }^{2, b}$, Liu Xingdong ${ }^{1, c}$ \\ ${ }^{1}$ Department of student affairs, Southwest Forestry University, Kunming 650224, China \\ ${ }^{2}$ School of Mechanic Engineering and Transportation, Southwest Forestry University, Kunming \\ 650224, China \\ alitianwei969@163.com, b27634347@qq.com, c295816986@qq.com
}

Keywords: internal working models; psychopathology; attachment

\begin{abstract}
Objective: Internal working models, as the important concept of attachment theory, can explain some psych-pathological diseases, the object relationship and therapist-client relationship background from the cognitive process view. Date Sources: The relevant articles were searched for in China Journal Full-text Database in China National Knowledge Infrastructure network with the key words "internal working models, attachment, psychopathology". Meanwhile, the relevant articles on internal working models and psychopathology were searched for in ProQuest Psychology Journals Database with the key words "internal working models, attachment". Besides, the relevant researches of 4 books on psychopathology were also consulted. Study Selection: The literatures were selected primary. The inclusion criteria were (1) those attachment on psychopathology and (2) those on the feature, developmental structure and attack mechanism of some psych-pathological symptom of internal working models. Exclusion criteria: repetitive researches were excluded. Data Extraction: There were 62 literatures in accordance with the above-mentioned criteria, and 16 were selected for analyzing after classifying and arranging. Articles with repetitive content and irritated topics were excluded. Data Synthesis: The internal working models influenced the formation mechanism of psych-pathological disease. As an interpretative filtration it could affect and guide cognitive-affective processes (attention, memory, expectation, and attributions, etc.), which could guide the behavior of individual to others, so as to influence the response manner of others to individual. On the contrary, the response manner of others had effects on internal working models. Meanwhile, the internal working models explained for therapist-client relationship and the object relationship background of the attack of psych-pathological diseases. Conclusion: Studies before pay attention to the influences on psych-pathological diseases from the emotional view, but ours pay attention to the great inspirations from attachment view so as to enrich the guiding theory of psych-pathological diseases treatment.
\end{abstract}

\section{Introduction}

As Arietta Slade said, Attachment theory provides a widely and profound perspective for researches on the social function of human being, it altered the view and response of which clinical pathologists hold on their patients, and it had a potential effect on dynamic theory of therapeutic relationship. As a very important concept of attachment theory, internal working models integrate cognition theory with emotional theory; it also has significance on how to explain the potential factors of some pathological mental deceases. We searched for the articles relevant with internal working models and psychopathology in China Journal Full-text Database in China National Knowledge Infrastructure network and in ProQuest Psychology Journals Database.

\section{The Origins of Internal Working Models and its' Intermediary role}

Clinical psychologists consider that people would be apt to dynamic reconstruct their unresolved relationships such as the relationship between their parents, sisters and significant others, and it 
become part of power which could influence the present relationship between their spouse, employers, colleagues and so on.

\subsection{The Origins of Internal Working Models}

At the beginning of the attachment theory founding, Bowlby thought that the children's attachment is a behavior control system which possesses target correction strategy, children can make use of the organization view to control their behavior, and deal with the relationship between his/her mother. The development of attachment behavior implicate four stages of control system, those are (1)No differences in the social response period (2)Selective social response period (3)Specific attachment period (4)Multiple attachment period. With the development of cognitive psychology, psychologists proposed many theories on representation, which can provide favorable foundation on understanding the development of attachment theory. The attachment development theory which Bowlby held turned to working models from control system, he made emphasis on the reconstruction of cognitive-emotion, and he thought that internal working models could alter with the different experiences although it has stability to some degree. Just as Bowlby draw support from the theory of control system which expanded the drive-reduction theory on maternal-infant affection Freud referred, the concept of internal working models proposed could expand the concept of cognitive representation, also it expanded the important psychoanalysis view about individual early experiences' influences on relationship development.

\subsection{Intermediary role of Internal Working Models}

Bowlby put forward the concept of internal working models clearly, in order to explain the process of internalization. Internal working models are the immanent action mechanism which can influence attachment relation development of individual. Bartholomew et.al. proposed a two-dimensional internal working models of adult attachment, in order to explain the cognitive mechanism of adult attachment. This model mainly includes self-cognition and others cognition, it was developed on the basis of childhood experience, and undertaking the task of organizing the attachment information at present. The four attachment styles have different internal working models: People of secure attachment type are thought to think about themselves and others with positive view; they will be happy to seek intimacy with others. In contrast, people of fearful attachment type think about themselves and others with a negative view, so expect the worst. Many people of anxious attachment type like others but their full of self-doubt, the result will depend too much on others, and people of detached attachment type like themselves but don't respect others.

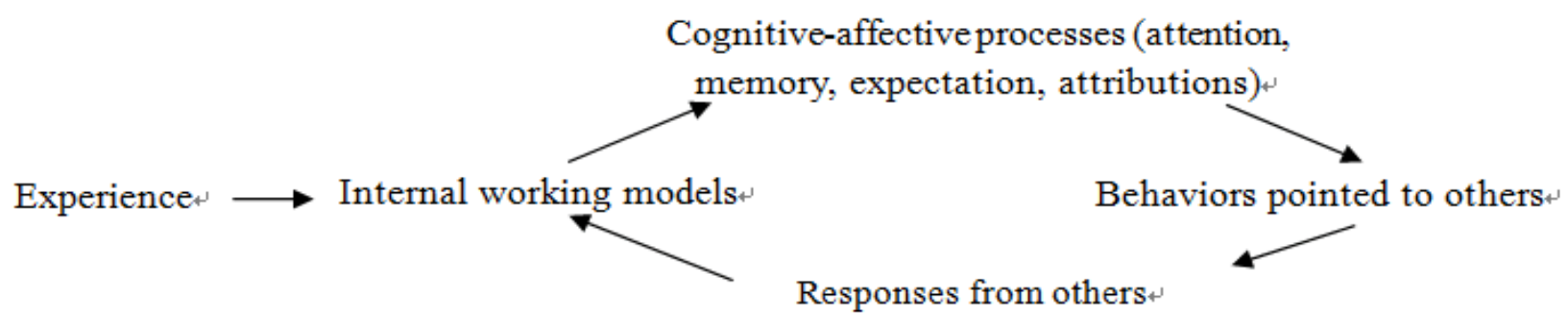

Fig.1 Intermediary role of internal working models in relation development

As illustrated in Fig.1, based on early attachment experiences with significant others, Internal working models as an explanatory filter can influence and guide the individual cognitive psychological process, such as attention, memory, expectation, and attribution and so on. This process can guide the behaviors what individual act to others, and then to influence others' response on individual and the responses from others in turn have effect on the internal working models. In this way, by constantly repeating strengthening, internal working models play a gradually automatic unconsciously role, it can guide the cognitive/emotional process, on the other hand, it could be influenced by the life events, internal working models play an intermediary role on the attachment relationship. For example, as such a stranger's glance, secure attachment type people would not give certain communication significance, but however, insecure attachment type people would attention and are apt to explain it according to their representation and to take appropriate action. 
The individual internal working models which on self, significant others, and the relationship between self and significant others, have important significance of adaptation, it can not only help individual expectant the future communication situations, but also can guide the individual to make a certain plan to cope with those situations. Therefore, to some degree, this intervening variable can be as explanatory factors to some psychiatric disorder.

\section{The Significance of Internal Working Models in Psychopathology}

Relationship is a double-edged sword. It is a basic characteristic of human being social society, although it is essential source of assistance, it also become potential sources of stress. Relationship between attachments is no exception. Experimental results show that separating young monkey from his mother by force, both the baby and mother show elevated levels of cortical, the stress activated their hypothalamus pituitary adrenal system, Immune function changes: lymphocyte reduce in vivo circulating, antibody sensitivity turn weaker. When mother and child reunion, their immune function returned to normal. Psychiatrist Bowlby just realized the importance of attachment in pathology, he thought that the personality development and emotional security of infant, related to the role or availability and quality of object for infant attachment. The destruction of early attachment will lead to children emotional crisis, and might show a sudden depression or anxiety state. Similarly, many psychopathological disorders due to the basic anxiety that fear of separation from the attachment object. During World War II, so many orphans who lost maternal love, lack of normal responses to environmental, showed severe mental disorders.

\subsection{Reactive Attachment Disorder}

Reactive attachment disorder also known as maternal deprivation syndrome is a kind of attachment behavior disorder caused by infant maternal deprivation. Causes of morbidity due to childhood rearing environment, such as maternal deprivation, replacement of caregivers, poor relationship in orphan, and their basic needs can not met. They were often suffered by parental indifference, neglect or abuse, and even intentional injury. More common in children who are raised in social welfare institutions or foster families. Patient children of two to five years old show that attachment behavior without choice to others, and they are apt to seek attention and make no difference friendly behavior, symptom relieve as the environment change. Often accompanied by difficulties with peer interaction, they can not establish close relationship and trust with peers.

As a result of the neglect or abuse parenting behavior, children have no safety or lack of trust, and because of their signals always are ignored or misunderstood, they might thought that themselves as troublesome. The representation children formed about his or others is negative, and it has relative stability, influencing the expectation of the future relation, furthermore, it becomes the model of foundation of relationship that is internal working model.

\subsection{Depression and Anxiety Disorder}

Psychoanalytic theorist emphasis early mental traumatic childhood, especially loss which play a pathogen role in mood disorders. If a person who lost his love, he would hold ambivalent attitude to his love, that is both love and hate, then negative emotions turned into intense anger, and positive emotion turned into guilt. Because of guilt, person concerned would anger toward himself, which leads to self-hatred and despair. In this case, a person would suffer from depression.

As mentioned above, the individual would be apt to explain life event and take appropriate action according to his/her internal working models. Therefore, there would be maladaptive vicious spiral model to the individual who have no security. That is negative emotion and cognition on themselves, negative expectation on others, and the self-defeating expectations in interpersonal relationships. Finally lead to pathological mental appearance. For example, a girl might think herself as unwelcome and offensive when she has a negative model. She might think that if she went to dancing party, no one would ask her to dance, and that young man invited her to dance but because of his poor to her. Like this, she would ever make such negative expectation and reaction in these situations, thus resulting in low self-esteem and depression. 
It is very important to make clear to the inner reflection way that patients with their significant others in clinical treatment. By education, we can enable patients to learn the nature and process of depression, encouraging patients to express their feelings better, communicating with others more closely, improving the negative representation and expectation of patients, in order to relieve symptoms.

\subsection{Enlightenment on Therapist-client Relationship}

In fact, there are two functions in attachment object for effective therapist: secure base and safe haven. As secure base, therapist should analyze if their relationship is useful to the patients, and as safe haven, therapist should provide an unconditional caring and help the patients to regulate negative emotions when they suffer a setback. Therapist should analyze why the reaction of patients below the ideal and acceptable manner, for example, why the patient A showed indifferent, but patient B showed honor and unconditional compliment. Therapist should make use of their knowledge to guide patient to self awareness, they might have the ability to distinguish the conscious or unconscious behavior of patient. Meanwhile, therapist should concern the transformation of internal working models what patients hold on therapeutic relationship along with the development of the treatment. Patients might continuously test the secure base or safe haven which therapist provided because of the early unfortunate attachment, so therapist must realize the potential significance.

\subsection{Object relations background which psychological pathological disorders occurred in}

Researches on the significance of interpersonal attachment have provided advanced support on object relations therapy for depression and other disorders treatment. Some previous empirical researches not make any hypothesis of the potential causes of pathological psychology, but to concentrate on the occurrence and persistence of the present symptom in object relation background. For example, comparing with normal person, the social support network of patients with depression, patients with eating disorder, patients with panic disorder, and patients with substance abuse, is narrower. They also have many apparent marital problems. Actually, if companion or parents are censorious and negative to patients with depression or patients with schizophrenia, their symptoms are more likely to relapse. Research by David showed that the individuals often have senses of alienation and intolerance when the family environment is too tight or too loose, thus increasing the risk of idioctonia. They have a great anxiety because they are afraid of abandoned by their significant others.

\section{Conclusion}

In addition to biological and genetic factors, not only internal working models can influence the formation mechanism of psychopathology diseases, it just discussed on the level of attachment representation. But, internal working models indeed help us to know part of internal mechanism in normal development, it has important significant on clinical diagnosis and family therapy.

\section{References}

[1] Waters E, Posada G, Crowell JA, etal. The Development of Attachment: From control system to working models. J Psychiatr 1994;57(1):32-56.

[2] Kahn WA, Kram KE. Authority at work: Internal models and their orgnizational consequences. Acad Manage Rev 1994;19(1):17-34.

[3] Borelli JL, David DH, Attachment theory and research as a guide to psychotherapy practice. J Imag Cognit Pers 2002;23(4):257-87.

[4] Cassidy J. Adult romantic attachment: a development perspective on individual differences. J Rev Gen Psychol 2000;4(2):111-31.

[5] Compas BE, Gotlib IH. Intrlduction to clinical psychology -Science and Practise. Translation by Yao Shu-qiao, Zhu Qiong-zhao. Beijing: People Hygiene Publishing House, 2004:281-8. 
